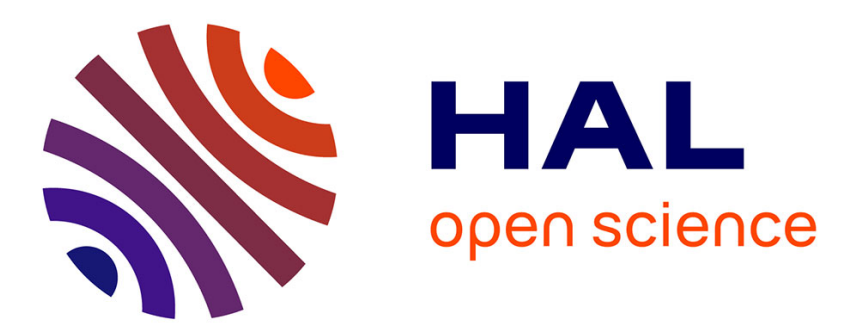

\title{
Entre réalisable et réalisé: DE et LIAO potentiels en chinois du nord
}

Christine Lamarre

\section{To cite this version:}

Christine Lamarre. Entre réalisable et réalisé : DE et LIAO potentiels en chinois du nord. Cahiers de linguistique - Asie Orientale, 1994, pp.155-167. 10.3406/clao.1994.1776 . hal-01492335

\section{HAL Id: hal-01492335 \\ https://hal-inalco.archives-ouvertes.fr/hal-01492335}

Submitted on 18 Mar 2017

HAL is a multi-disciplinary open access archive for the deposit and dissemination of scientific research documents, whether they are published or not. The documents may come from teaching and research institutions in France or abroad, or from public or private research centers.
L'archive ouverte pluridisciplinaire HAL, est destinée au dépôt et à la diffusion de documents scientifiques de niveau recherche, publiés ou non, émanant des établissements d'enseignement et de recherche français ou étrangers, des laboratoires publics ou privés. 


\title{
Entre réalisable et réalisé : de et liao potentiels en chinois du nord
}

\section{Christine Lamarre}

\section{Résumé}

This paper gives a tentative interpretation of the double value (perfective and potential) of two forms, de and liao, in some Northern Mandarin dialects. The potential suffix liao has not been discussed up to now, and we claim that the link we can see between its potential and perfective values can also be seen for de. The hypothesis of a haplology to account for potential $V$-de or V-liao forms is also discussed.

\begin{abstract}
This paper gives a tentative interpretation of the double value (perfective and potential) of two forms, de and liao, in some Northern Mandarin dialects. The potential suffix liao has not been discussed up to now, and we claim that the link we can see between its potential and perfective values can also be seen for de. The hypothesis of a haplology to account for potential $V$-de or V-liao forms is also discussed.
\end{abstract}

\section{Citer ce document / Cite this document :}

Lamarre Christine. Entre réalisable et réalisé : de et liao potentiels en chinois du nord. In: Cahiers de linguistique - Asie orientale, vol. 23, 1994. pp. 155-167;

doi : $10.3406 /$ clao.1994.1776

http://www.persee.fr/doc/clao_0153-3320_1994_num_23_1_1776

Document généré le 02/06/2016 


\title{
Entre réalisable et réalisé : de et liao potentiels en chinois du nord
}

\author{
Christine LAMARRE
}

Nous nous proposons ici d'examiner quelques problèmes théoriques posés par l'emploi dans certains dialectes du nord de la Chine d'un morphème LIAO dans les formes potentielles des combinaisons résultatives, ex. 我拿動了 (= Putonghua 我拿得動 wó nádedòng "j'arrive à soulever"). Notre réflexion portera surtout sur les diverses facettes du parallélisme --- déjà relevé par Dragunov 1958:129-30 --- présenté par DE et LIAO, qui peuvent dans certaines conditions conférer au verbe qu'ils suivent la valeur de "réalisé" et celle de "possible".

Les monographies décrivant les dialectes du Shanxi et du Shandong publiées au cours de ces dernières années nous ont permis de vérifier l'étendue d'un phénomène grammatical sans doute propre aux dialectes du nord, dont deux manuels de mandarin du nord (L. Wieger 1912 pour Hejianfu = Xianxian ; J. Mullie 1932-37 pour le Jehol $=$ Chengde) donnaient déjà une description détaillée au début du siècle. Ces deux localités, ainsi que Changli qui présente un système similaire décrit en 1960, sont situées dans l'actuel Hebei. Dans Lamarre 1994, nous donnons une description du fonctionnement de ce LIAO à partir des données disponibles, complétées par une brève enquête personnelle au Shanxi et au Shandong. La carte et le tableau ci-après donnent un aperçu de la distribution géographique et des formes phonétiques prises par ce $L I A O$. 


\section{LES PARADIGMES DE LIAO ET DE EN PUTONGHUA (PTH)}

1. Complément résultatif C (ex. 看星了, 秵得飽, 打不死 ).

Si à l'origine $D E$ et $L I A O$ étaient tous deux compléments résultatifs (cf. Liu Jian 1992 etc.), telle n'est pas leur fonction principale aujourd'hui en langue standard.

$D E$ ([dé]) a en pékinois le sens de "finir" (cf. Chao 1968).

$L I A O$ : tonique [liăo], il indique, dans sa forme potentielle essentiellement, que l'action peut s'accomplir jusqu'au bout, ex. 太多, 我贸不了 "il y en a trop, je ne pourrai pas tout manger". Sur l'existence d'un complément [lou $\left.{ }^{\circ}\right]$ en pékinois, cf. Ma Xiwen 1983.

\section{Marque de l'aspect accompli [V-]}

$D E$ : très fréquent des Tang aux Ming, obsolète en $\mathrm{PTH}$, se trouve "fossilisé" comme suffixe non productif dans des verbes comme記得 "se rappeler", 惩得 "(re)connaître" etc., Cf. Jakhontov 1957/1987:164.

$L I A O$ marque en PTH l'aspect accompli sous sa forme atone $L E$.

3. Support "vide" de la forme potentielle (le "dummy potential complement" de Chao 1968). Apparaît en [ V 得 ] / [ V 不 ].

Dans la mesure où le passage de 1. à 3. implique la spécialisation de certains éléments du paradigme des $\mathrm{C}$, une perte de sens lexical, et un élargissement de la "combinabilité" (ce que J. Matisoff appelle "juxtapository productivity") avec les verbes $\mathrm{V}$, il s'agit bien d'une grammaticalisation.

$D E$ indique que l'action est (in)appropriée, et aussi une (im)possibilité passive, tenant à une propriété spécifique au patient de V) ex.：道果子缱生，装不得 "ce fruit est encore vert, il n'est pas comestible"), cf. Liu Yuehua 1980, Jakhontov 1957, Lü Shuxiang 1980). (Sur l'analyse de la contrepartie affirmative 契得 en 得 [*得 ], cf. notre $\S 2$ sur l'haplologie.) Le refus de Chao 1968:454 d'admettre ce sens "passif" de la valeur potentielle de $D E$ tient sans doute à sa volonté d'englober les dialectes du nord et du sud dans un même système, mais son argument (les verbes 記得, 羿得, 暁得 ) méconnaît la nature du suffixe $-D E$ (figée) dans ces verbes en langue standard.

$L I A O$, sous sa forme tonique [liăo], indique une (im)possibilité active, tenant aux circonstances entourant l'agent de V. Il perd donc sa valeur lexicale de "finir" et n'exprime plus qu'un potentiel "actif" (cf. Liu Yuehua 1980), ex. 明天我没事, 来得 了. Il semblerait (Ota 1965) que cet usage de LIAO soit plutôt septentrional et corresponde parfois à un 掉 [diào] en mandarin du sud.

A ce niveau 3,DE et $L I A O$ ne s'emploient que dans les formes potentielles. ZHAO 着 connaît le même type de vidage dans 用不着 yòngbuzháo, "il est inutile de" (équivalent de 用不了 yòngbuliăo). 


\section{Affixe dans les formes potentielles de V C : V 得 $\mathrm{C} \sim \mathrm{V} C 了 / \mathrm{V}$ 不 $\mathrm{C}$}

En PTH, c'est $D E$ qui fonctionne comme infixe dans les formes potentielles du type 看得見/看不見,開得開/開不開,拿得動/拿不動,去得了/去 不了 etc.; où il se trouve en apparente symétrie avec la négation 不 $B U$, qui exprime elle l'impossibilité en $\mathrm{V}$ 不 $\mathrm{C}$.

Or de nombreux dialectes du nord utilisent eux au niveau 4 un morphème $L I A O$ (cf. tableau et carte), non comme infixe mais comme suffixe, brisant ainsi cette symétrie avec $B U$. On a alors \{看見工／看不見\}，\{開開了/開不開\}，\{拿動了 拿不動 \}, \{去了工/去不了\}etc...

Il est à noter que quand $D E$ ou $L I A O$ changent ainsi de paradigme, l'opposition entre potentiel actif/passif qui les caractérise au niveau 3 (ceci aussi bien en PTH que dans les dialectes à $L I A O$ potentiel que nous avons examinés) se neutralise, \{看見而\} correspondant au 看得見 du PTH. Le passage de 3 à 4 représente donc bien celui à un stade ultérieur de grammaticalisation, du point de vue de la perte de sens lexical comme de celui du changement de paradigme.

Il semble qu'à Yanshan (sud de Tianjin) 着 [tsau ${ }^{\circ}$ ait connu un "vidage" similaire, ex. : 拿動着渓? -- 拿動着 (=PTH 能拿動 ). Le même 着 ${ }^{\circ}$ y marquerait aussi l'accompli ( 《塩山県志》: 899)...

\section{SYMETRIE DES PARADIGMES ET HAPLOLOGIE}

\subsection{Zones où $D E$ marque le potentiel au niveau $4: \mathrm{V}$ 得 $\mathrm{C} / \mathrm{V}$ 不 $\mathrm{C}$}

Dans la zone où $D E$ fonctionne au niveau 4 (la langue standard PTH), LIAO reste au niveau 3, dans le paradigme des $C(V$ 得了 $/ V$ 不了) où il exprime le potentiel actif. Quand le $D E$ du niveau 3, exprimant le potentiel passif, rencontre son homonyme du niveau 4 dans une forme potentielle $\mathrm{V}$ 得得 $/ \mathrm{V}$ 不得, on a une haplologie qui produit V 得 . (Cf. Chao 1968:247 à propos de $L E 1$ et $L E 2$ en PTH: "In such cases Mandarin always avoids a repetition of the same syllable by way of haplology : -le le $\rightarrow-l e . . . "$; cf. aussi p. 298 à propos de 的 ).

Hu 1981 fait en effet remarquer l'existence à Wenshui (Shanxi) de compléments potentiels comprenant un $D E$ redoublé (ex. 果子逞青，照不的／ 果子紅了, 典的的了, 的=得). Il analyse le premier comme correspondant au 得 $\mathrm{du}$ complément potentiel pékinois păode kuài "courir vite" et le second comme le 得 $D E$ de 使得 ou 去不得. I I en conclut enfin que des formes comme 契得 en PTH doivent s'analyser comme la réduction par haplologie de 貫得得, ce qu'il représente par $\mathrm{V}$ 得 [ $*$ 得 ]

Ceci nous semble étayer l'intuition de $\mathrm{Lu}$ Zhiwei et Zhu Dexi pour qui $\mathrm{V}$ 得， en tant que contrepartie dissymétrique de $\mathrm{V}$ 不得, doit s'analyser en $\mathrm{V}$ 得得 $\quad \mathrm{Lu}$ 1957:79：『影然我们不能期望有一個“慗得得”』; 
Zhu 1982:133『“説得”実際上応該分析為 “説得得”，前一個 “得”是助詞， 和“看得見”裹的“得”相当. 後一個“得”是充任補語的動詞, 和“看得見” 裹的 “見”相当. 只是因為雨個 “得”語音形式相同,所以把助詞 “得” 略去了.」. Li Xiaoqi 1985 approuve cette analyse.

\subsection{Zones où $L I A O$ marque le potentiel au niveau $4: \mathrm{V} \mathrm{CI/V}$}

Dans les zones où c'est $L I A O$ qui fonctionne comme marqueur potentiel du niveau 4, c'est $D E$ qui est parfois réduit au niveau 3 , où il fonctionne dans le paradigme des compléments résultatifs $C$. En effet, pour certains dialectes de cette zone qui n'admettent pas la forme V-DE pour exprimer le potentiel passif, ce dernier se rend à l'aide d'un verbe auxiliaire préverbal, ex. 能 $\mathrm{V}$ etc... Mais parmi ceux où V-DE possède ce sens, certains, comme Changli ou Zunhua (Hebei), Fenyang et Wuxiang (Shanxi), l'utilisent nettement dans le cadre de la forme potentielle en V 3 I $\mathrm{V}$ 不 (cf. § 3).

De même, au Shanxi, le suffixe figé $D E$ qui se trouve dans les verbes 記得， "se souvenir", 琶得 "connaître" et parfois 暁得 "comprendre", fonctionne souvent comme un C (la négation obligatoirement en $V$ 不得 laisse supposer une lexicalisation moindre qu'en PTH), et la forme affirmative doit forcément comporter LIAO pour nos informateurs de Yuxian, Qinxian, Changzhi, Lingchuan, et Linxian (les verbes concernés peuvent eux varier), ex. 我琶得了/惒不得. En cas d'objet celui-ci se place entre $D E$ et $L I A O$, comme à Changli, ex.

Changzhi 我珰得他了 "je le connais" / 涊不得他.

Changli 他病好咧, 契得干飯 liou $^{\circ}$ "Il va mieux, il peut manger du riz normalement". $/$ 照不得干飯.

Quand le $L I A O$ du niveau 3, exprimant le potentiel actif, se combine avec le marqueur $L I A O$ du niveau 4 , on peut avoir soit un $L I A O$ obligatoirement redoublé, ex. Lijin au Shandong ou Changzhi au Shanxi :
Lijin
去哩哩（＊去哩）／去不了
上去哩／上不去
Changzhi
回了了（*回了）/回不了
回去了/回不去

soit un deuxième $L I A O$ facultatif (Pingyao, Lingchuan etc.), soit un seul LIAO (Shandong Boshan). Tout ceci suggère une haplologie en cours dans certaines régions, d'autant plus que pour les locuteurs admettant $\mathrm{V} L I A O$ et $\mathrm{V}$ LIAOLIAO, ce dernier est utilisé par les personnes plus âgées (Hou 1983 pour Pingyao, nous avons obtenu les mêmes commentaires pour Taiyuan). L'interprétation de formes comme V LIAO au Shanxi ou au Shandong et V DE en PTH comme provenant par haplologie de V LIAOLIAO et V DEDE permet de rendre compte du fait qu'elles subissent les mêmes contraintes sémantiques que le $L I A O$ et le $D E$ du niveau 3 en ce qui concerne le sens actif/passif de la possibilité.

De fait V了了évolue parfois en un équivalent de 会 V "savoir V", summum de la possibilité active, c'est-à-dire qu'au lieu d'exprimer la possibilité de l'action en 
se référant à une situation donnée (ex. 明天 我有事兒,来不了 "Demain j'ai à faire, je ne peux pas venir"), il se réfêre aux capacités de l'actant. Ainsi à Changzhi (Shanxi), on a 説了了 au sens de "(cet enfant) sait parler". A Lingchuan, 他看了了 équivaut au PTH 他看得懂 "lui, il a étudié le français, il peut lire -- et comprendre -- ce livre (écrit en français)". Et à Lucheng on a 我打了了 pour "Je sais jouer (au pingpong). L'impossibilité correspondante s'exprime par V 不了. Parfois le complément s'insère entre les deux LIAO ex. Lingchuan 他喝了酒了 "il peut boire (sans que l'alcool lui tourne la tête" / 喝不了酒 . (le deuxième 了 est prononcé /lou/ comme $L E 1$ et non $/ \mathrm{la}$ / qui correspond à $L E 2$, cf. tableau); ou Changzhi : 我騎了馬了 $=$ PTH 我会䭲馬 "je sais monter à cheval" / 踦不了馬.

L'ordre des constituants est donc V了 O J/V 不了 O.

Nous donnons ci-dessous une représentation schématique du processus de grammaticalisation de $D E$ et $L I A O$. Les formes entre \{ \} sont celles de certains dialectes à $L I A O$ potentiel quand elles diffèrent du PTH ; la prononciation [ ] représente celle de la langue standard.

Niveau 0 : verbe

$L I A O$ 了 [liăo] "achever"

$D E$ 得 [dé] "obtenir"

passage à la position postverbale

NIVEAU 1 : complément résultatif

LIAO 三天里做不了 [liăo]

$D E$ 做不得 [dé]

$=$ impossible de $\left[\begin{array}{ll}\downarrow \\ \downarrow\end{array}\right]$ finir en trois jours perte de sens lexical

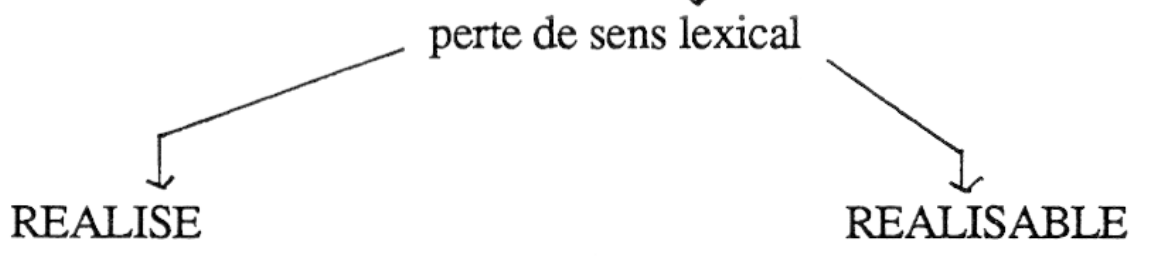

NIVEAU 2 NIVEAU 3 "dummy potential complement" $L I A O$ 我喝及 [le] 両杯. LIAO, pot. actif $\{$ 来了了\}/\{来不了\}

$D E$ (obsolete,

figé dans

来得了／来不了

記得 jide, 認得 rende) ou dans $\{$ 恋得 $(0)\}\}$ ～～認不得 $(0)\}$

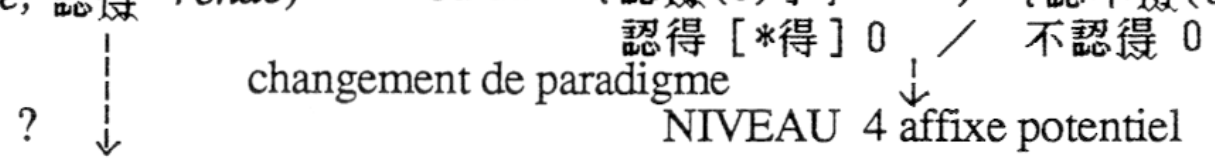

$L I A O$ 我看見了一個朋友

$D E$ 他唱得好聴
\{看見了／看不見\}

看得見 / 看不見 
Les autres systèmes existant en Chine du Nord (LIAO et $D E$ de niveau 4 coexistants, $D E$ préverbal, $N E N G$ V C etc.) devront faire l'objet d'une autre étude, les données manquant pour le moment.

\section{ASPECT ET MODE}

Jakhontov (1957, 1987:195) soulignait que la catégorie du temps et de l'aspect en chinois correspondait parfois à des valeurs identiques à celles qu'exprime le mode dans d'autres langues. Comme nous l'avons vu en 2.2 , il y a des dialectes comme Changli (Hebei) où le 得 $D E$ du potentiel passif fonctionne au niveau 3 et a besoin du $L I A O$ du niveau 4 pour se réaliser. Dans d'autres dialectes encore, ce $D E$ est remplacé par un autre complément $C$ grammaticalisé au niveau 3 , comme par ex.

成 à Pinyao ou à Jiexiu (Shanxi) qui exprime aussi le potentiel passif (ce que Hou Jingyi 1982 exprime par" 可否 " par opposition au potentiel actif de $L I A O$ " 能否". Il remarque aussi que 成 a dans ce cas sa prononciation populaire, signe fréquent de grammaticalisation). A Pingdu (Shandong), on trouve un 着 $Z H E$ qui exprime certaines valeurs de potentiel passif rendues ailleurs par $D E$ : "valoir le coup d'acheter, être rentable". Tous ces morphèmes de niveau 3 apparaissent ainsi dans l'environnement $\mathrm{V} \sim \mathrm{J} / \mathrm{V}$ 不 $\sim$, ex. :

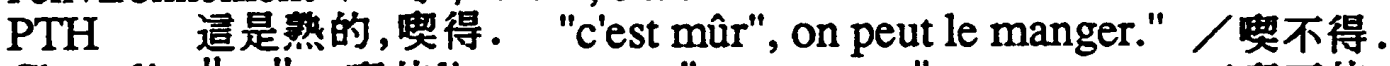

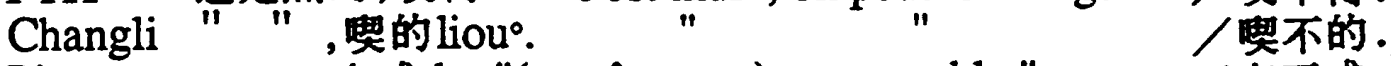

Pingyao 穿成 lo. "(ce vêtement) est mettable." / /

Pingdu 買着了. "tu peux l'acheter $\rightarrow$ C'est une occase, ce n'est pas trop cher." 買 [ $\left[\mathrm{ts}^{\circ} 0^{\circ} \mathrm{\partial}^{\circ}\right.$ ] ／買不 [tșua53]. Cf. Chengde 他那個買賣做的做不的 - 做的. (Mullie 1937:67) "Is his trade profitable? --.- Yes, certainly".

et Dezhou：這個活兒干不的. "Ne fais pas ça, c'est trop mal payé".

Ainsi, l'idée, qui peut sembler bizarre à priori, qu'un membre du paradigme des compléments résultatifs exprime en tant que "résultatif" une valeur de "potentiel", semble pourtant se vérifier et pour $D E$ (possibilité passive), et pour LIAO (possibilité active dans 去不了 en Putonghua), et pour d'autres C, éléments exprimant un certain type de potentiel passif dans d'autres dialectes. Tous ces compléments $\mathrm{C}$ fonctionnent au niveau 3 , dans les formes potentielles.

Un dernier argument, venant du sud celui-là. A Wenzhou (Zhejiang), il semble que le passage du niveau 3 au niveau 4 n'ait pas eu lieu. La seule combinaison V C est porteuse en puissance de sens potentiel, et s'oppose à V 不 C. YOU Rujie 1980 remarque en effet que ni "得 " $D E$ ni aucun autre élément n'est susceptible de s'insérer entre $\mathrm{V}$ et $\mathrm{C}$. La contrepartie positive de 哭不完 est donc

契完, (au sens de "pouvoir manger en entier"), alors même qu'il existe une forme " 講得/講勿得 ". You interprète ce $D E$-là comme étant resté à un stade de développement moindre qu'en PTH, n'étant pas parvenu à l'étape du passage au sens potentiel (ce que nous nommons ici niveau 4). 
On trouve apparemment à Yanchuan (Est du Shaanxi) et Fenyang (Ouest du Shanxi) un $D E$ postverbal indiquant le devoir.

\section{PARALLELISME $L I A O \sim D E$ :LEVOLUTION SEMANTIQUE}

\subsection{Le lien entre les niveaux 2 et 3 : entre suffixes aspectuels et "dummy potential complements"}

Le lien existant en pékinois entre le suffixe aspectuel $L E$ et le faux complément résultatif $L I A O$ est masqué par l'atonie et l'amuissement de la voyelle du premier, mais comme le souligne A. Rygaloff 1972:187 à propos des valeurs aspectuelles de certains résultatifs en pékinois (zháo 着, zhù 住, jiàn 見, kāi 開， shang 上, etc.), "ces valeurs, dans la plupart des cas, ne sont que des spécifications de la valeur d'accompli simple, et celle-ci, à la vérité, est résultative également : dans les mêmes limites que le suffixe -le, jouent en effet des couples de formes potentielles en -liăo, par exemple chi-bu/de-liǎo <ne pas / arriver à manger jusqu'au bout> -- qui non seulement s'expliquent par la valeur de ce suffixe, mais où se retrouve aussi la forme du verbe liáo <achever> dont ce suffixe provient."

La grammaticalisation des $C$ de niveau 1 au niveau 3 , où ils deviennent de simples supports de la forme potentielle, et ne conservent qu'un minimum de sens lexical (possibilité active/passive etc.) dans l'environnement \{V C $了 / \mathrm{V}$ 不 C $\}$, ou encore $\mathrm{V}$ 得 $\mathrm{C} / \mathrm{V}$ 不 $\mathrm{C}$, peut être considérée comme le pendant "réalisable" de leur grammaticalisation dans l'environnement $\mathrm{V} \sim$, où ils expriment le réalisé (l'accompli, ou le duratif pour 着 ). La complémentarité de leur distribution est masquée en PTH par la dissociation de leurs phonies due à l'atonie, mais le Vli॰ li॰ / V 不 lio de Lijin ou le $\mathrm{V} \operatorname{tss} l^{\circ} 10^{\circ} / \mathrm{V}$ 不 tşuə de Pingdu laissent entrevoir d'autres perspectives.

Peut-on donc parler d'une façon plus générale de corrélation entre suffixes marquant le réalisé et "dummy potential complements" ? Cette question mérite notre attention, comme en témoigne le cas de DAO (souvent écrit 倒 pour le distinguer de son homologue 到 au ton partant) qui à Hong'an (N.E. du Hubei) possède une valeur très étendue de réalisation (niveau 2) et sert de support aux formes potentielles avec un sens actif (niveau 3), par ex. 芳芳打得毛衣倒 = PTH 芳芳会打毛衣 "Fangfang sait tricoter" ( 《紅安県志:679》). A Daguan (Yunnan) aussi DAO a ces deux mêmes valeurs, ex. 去不去得倒? = PTH 能不能去 ? (《大関方言志》 p. 238). A Xining, on trouve $下 / x a /$ avec les valeurs d'accompli et de support de la forme potentielle également ( 《西案方言志》).

\section{CONCLUSION}

Un mot pour terminer sur la dissymétrie formelle de $\mathrm{V}$ 得 $\mathrm{C}$ et $\{\mathrm{VC}\}$ \}. La différence de position de $D E$ et $L I A O$ au niveau 4 prouve bien que l'on a affaire à deux systèmes distincts et non à un simple remplacement lexical. On peut sans doute l'expliquer par les autres fonctions assumées par $D E$ et $L I A O$ (cf. la représentation schématique à la fin du § 2). Rappelons que la symétrie V 得 $\mathrm{C} / \mathrm{V}$ 不 $\mathrm{C}$ 
n'est qu'apparente, aussi bien en diachronie (cf. Ota 1958: $§ 16.6 .3$ ) -- comme nous le signale la position de l'objet en Hakka par ex. : V 得 O 倒 / V O Nég. 倒 --, qu'en synchronie (Lin Tao 1957 se fonde sur la réalisation du ton léger en pékinois pour affirmer que 得 $-D E$ se rattache structurellement au verbe qu'il suit et 不-BU au complément qu'il précède). Le fait que le cantonais puisse placer la négation avant $\mathrm{V}$

得 C, et que comme Changsha (Zhang 1985), Daguan (Yunnan) et de nombreux autres dialectes méridionaux, il utilise V-DE avec un sens de potentiel actif, tout ceci montre bien la différence de système avec les dialectes du nord.

Un autre aspect du parallélisme $D E \sim L I A O$, celui de l'ambiguitté formelle, n'a pu être traité ici. Bien sûr, comme le note la description de Changli (Ding Shengshu), l'ambiguiité du complément en $D E$ (PTH 説得快 ) est levée dans ce type de dialectes ( 説快了 y est potentiel et 説得快 descriptif). Mais Jakhontov remarquait $(1957,1987: 249)$ que l'emploi du $了$ LIAO potentiel décrit par Wieger ou Mullie était impossible dans la langue écrite contemporaine en raison du risque de confusion avec le 了 $L E$ de l'accompli. De fait, dans la langue parlée, le risque d'ambiguitté semble assez faible, en ce qui concerne la plupart des dialectes que nous avons examinés tout au moins. Ceci tient à diverses raisons dont la différence phonétique entre $L E 1 \quad(\mathrm{~V} \sim \mathrm{O})$ et $L E 2$ ( $\mathrm{V} \mathrm{O} \sim$, cf. tableau) et la position de l'objet entre $\mathrm{C}$ et $L I A O$.

Enfin, mais c'est là loin d'être un point de détail, l'apparent isolement (sur la carte au moins) du pékinois prête à de multiples hypothèses. On peut penser bien sûr à des raisons internes, liées à l'amuissement de la voyelle de $了$ (/iau/>la/aussi bien pour $L E 1$ que pour $L E 2$ ), mais aussi à des raisons externes, "institutionnelles", tenant au rôle de capitale de Pékin : le poids du "mandarin" en tant que langue nationale aurait amené le pékinois à incorporer des traits propres au mandarin du sud (Nankin, cf. Rygaloff 1973:8). Une fois que le complément potentiel fera l'objet de toute l'attention qu'il mérite au cours des enquêtes sur le terrain, ces interrogations trouveront peut-être leur réponse.

Christine LAMARRE

Université de Jeunes Filles

d'Osaka

590 Sakaïshi, Daisancho 2-1

JAPON 


\section{REFERENCES GENERALES}

DRAGUNOV A. (1958). Long Guofu. 《現代漢語語法研究》科学出殿社.

JAKHONTOV S. (1957). Categoria glagola v kitaickom iazike. Traduction chinoise 1958.《現代漢語的動詞籍礂》 Beijing : Zhonghua Shuju ; Trad. japonaise de Mantaro Hashimoto：1987. 《中国語動詞の研究》Tokyo : Hakuteisha.

LAMARRE C. (1994). "Potential suffix LIAO in Northern Mandarin", Current Issues in Sino-Tibetan Linguistics, $\mathrm{H}$. Kitamura et al. ed., Osaka: The Organizing Committee of the 26th International Conference on Sino-Tibetan Languages and Linguistics. Pp. 210-19.

LI Xiaoqi (1985). 《関於能性補語式中的語素 “得” 〉《語文研究》四.pp. 11-18.

LIN Tao (1957). 〈現代漢語補足語里的軽声所反映出来的語法和語義問題〉

《北京大学学報》第二期. In 《現代漢語補語研究資料》 pp. 36-55.

北京語言学院語言教学研究所編, 1992. 北京語言学院出肘社.

LIU Jian et al. (1992). 《近代漢語虚詞研究》北京:語文出版社.

LIU Yuehua (1980). 〈可能補語用法的研究〉《中国語文》4, pp. 246-57.

LU Zhiwei (1957). 《漢語的構詞法》北京:科学出版社.

LÜ Shuxiang ed. (1980). 《現代漢語八百詞》北京:商務印書館.

MA Xiwen (1983). 〈関於動詞 “了”的弱化形式／・10u／〉《中国語言学報》， pp. 1-14. 北京:商務印書館.

OTA Tatsuo 太田辰夫 (1958). 《中国語歴史文法》Tokyo : Konan Shoin. (1965). 〈北京語の文法特点〉 《神戸外大諭箃》6月号 pp. 37-55.

RYGALOFF A. (1973). Grammaire élémentaire du chinois. Paris : Presses Universitaires de France.

ZHU Dexi 朱徑熙 (1982). 《語法講義》北京: 商務印書館. 


\section{LISTE DES REFERENCES CONCERNANT DES DIALECTES PARTICULIERS}

$\left(^{*}\right)$ indique les dialectes pour lesquels un entretien avec un/des informateurs a eu lieu. Seuls les ouvrages contenant des descriptions du LIAO potentiel sont mentionnés. Les monographies 《方言志》sont désignées seulement par l'auteur et l'année de parution. Le lecteur est prié de se reporter à Lamarre 1994 pour les références complètes des données utilisées dans la carte et le tableau.

QINGHAI : Xining 張成才 1987. SHAANXI : Yanchuan 張崇 1990.

\section{SHANXI :}

《方言志》 : * Changzhi 侯精一 1985 ; Heshun 田希誠 1987; Jiexiu 張益梅 1991 ; * Lingchuan 金夢茵 1983 ;* Linxian 李小平 1991 ; * Pingyao 侯精 1982 ; Qixian 楊述祖，王艾録；* Qinxian 張振鐸 $1990 ; *$ Qingxu 潘䊒 武 1990 ; Shouyang 趋秉访族 1984;* Taigu㛫述祖 1983; Taiyuan : informateurs； Wenshui 胡双宝 1988; Wuxiang 史素芬,李奇 1990 ; Xiangyang 陳洞闲， 李唯実 1984 ; * Yuxian 宋欣檑 1991.

楊象掌 1992. 〈繁峙方言的 “的” 〉《山西大学学報》1, pp. 15-18.

宋秀令 1988. 〈汾陽方言中的“的” 〉《語文研究》2, pp. 60-62.

潘家懿等 1990. 〈河津方言述略〉《語言学論文集》 Taiyuan, pp. 33-42.

王偉 等1990. 〈㯰関方言的語法特点〉《語言学論文集》pp. 203-7.

徐祖明、王李金 1990.〈潞城方言的語法現象〉《語言学論文集》山西人民出

版社、太原. pp. 197-202*

侯精一 1982.〈平道方言動補式〉《語文研究》2,pp. 119-127.

胡双宝 1981 〈文水話的若干語法現象〉《語文研究》2,pp. 128-37.

HEBEI : Changli 中国科学院語言研究所 1960 ; Huolu(Huailu) 陳淑静 1990; 《県志》 :Zunhua 道化 1990 ; Yanshan 塩山 1991.

MULLIE J. The Structural Principles of the Chinese Language (Northern Pekingese Dialect. 1932 : vol. I, Peiping : the Bureau of Engraving and Printing. 566 p; 1937 : vol. II \& III, Pei-P'ing: Pei-t'ang Lazarist Press. 691 p.

WIEGER L. (1912). Chinois Parlé - MANUEL - koan-hoa du Nord, non-pékinois. $3 \mathrm{e}$ éd. refondue. $1146 \mathrm{p}$. 
HENAN : 《方言志》: Zhengzhou 虚甲文 1992 ;

《目志》: Anyang $1990 ;$ Tangyin 1987.

賀媳 1989 《獾嘉方言研究》北京:商務印書館.

賀蘶 1981 《済源方言記略》《方言》第一期 pp. 5-26.

\section{SHANDONG}

《方言志》: * Dezhou 曹延杰 $1991 ;$ Jimo 趋日新 等 $1991 ; *$ Lijin 楊秋沢 $1990 ; *$ Linqing 張鸿垄 $1990 ; *$ Pingdu 于克人 1992 ;

Pingyi 李洪延 1987; Yexian 銭曾怡等 à paraître;

Zhucheng 諸城 : cf. 銭曾怡 等 1992 《濰坊方言志》.

《県志》: Feicheng 1992 ; Qingzhou : 1989 = 益都; Tengxian : 1990 ; Yanggu : 1991 ; Linju.

高文達 1988〈済南方言的語法特点〉《語言学通訊》第11期,pp. 1-9. Jinan.

Jining : HESSER J. (1905). Chinesische Grammatik Jentschoufu : Druck der Katholischen Mission. 241 p.

Liaocheng：張鶴泉 1988. 〈魯西方言中的語気助詞“喽” 〈《語言学通訊》 第11期, pp.93-98. Jinan：山東省語言学会.

*Boshan 博山 : informateur ; * Shanxian : informatrice.

高慎貴 1984. 《新泰方言研究》勝利油田教育学院 (non publié).

曹志耘 1988. 〈沂水方言的語法特点〉《語言学通訊》11, pp. 212-20. Jinan.

銭曾怡等 1992.《山東諸城方言的語法特点〉《中国語文》1, pp. 52-55

YUNNAN：張映庚 1990.《大関方言志》北京:語文出版社.

HUBEI : 《紅安県志》1992，上海人民出版社.

HUNAN 張大旗 1985. 〈長沙話 “得” 字研究〉《方言》第 1 期, pp. 46-63. ZHEJANG 游汝杰 1980.〈温州方言的語法特点及其歴史淵源〉《復旦学報》 増刊, 語言文字専輯, pp. 107-123. Shanghai. 


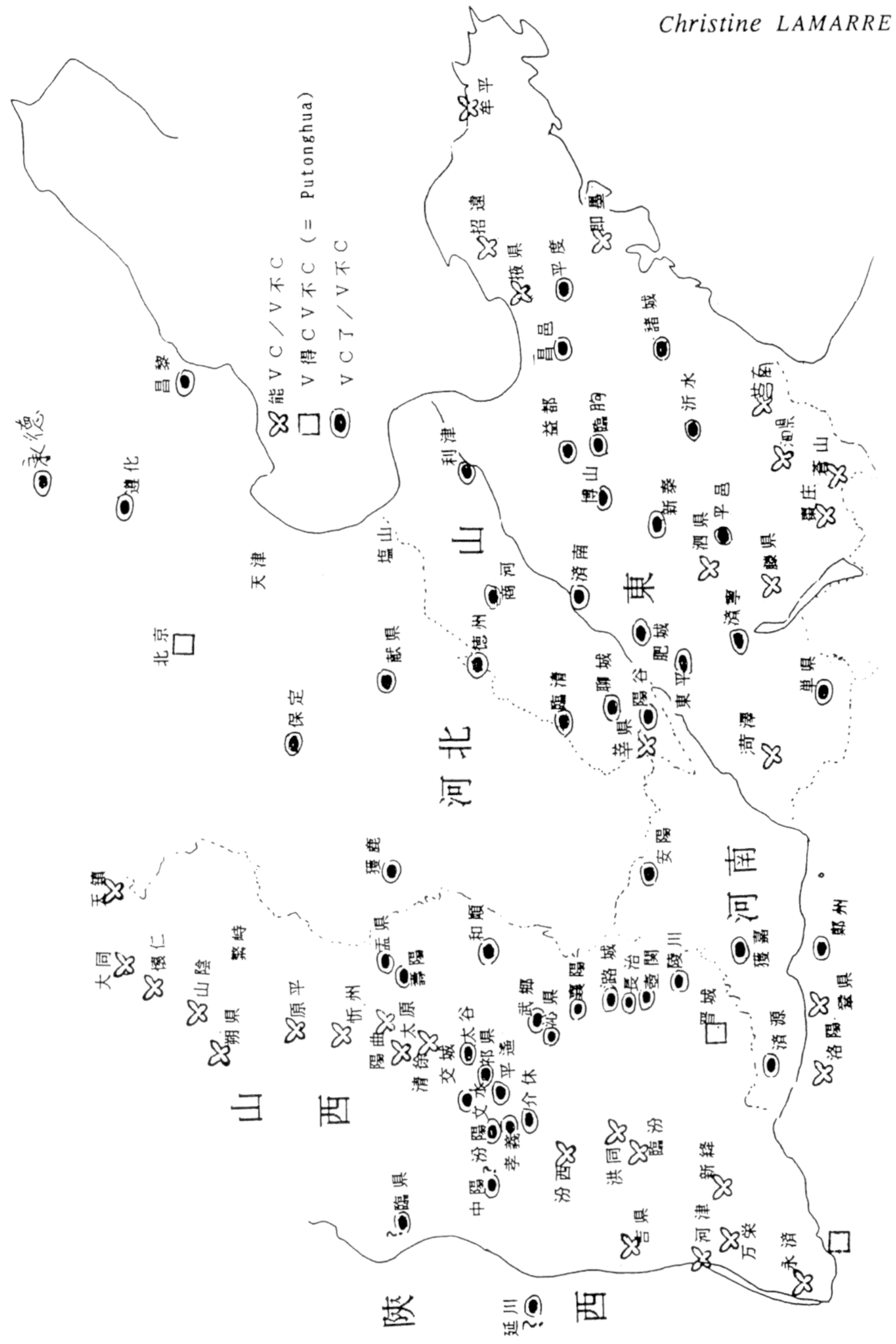


TABLE: DIALECTES UTILISANT LE LIAO POTENTIEL

\begin{tabular}{|c|c|c|c|c|c|c|}
\hline exemples & 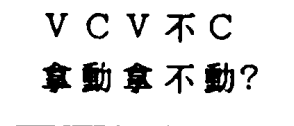 & $\begin{array}{c}\mathrm{V} C \mathrm{~J} \\
\text { 我拿動了 }\end{array}$ & $\begin{array}{l}\mathrm{V} 了(了) \\
\text { 他来了（了） }\end{array}$ & $\begin{array}{l}\mathrm{V} \text { 不了 } \\
\text { 来不工 }\end{array}$ & $\begin{array}{l}\text { LE1 } \\
\text { 咊工飯 }\end{array}$ & $\begin{array}{l}\text { LE2 } \\
\text { 他走 }\end{array}$ \\
\hline Linxian & $\mathrm{VCla}^{\circ}$ 不 & $\mathrm{VCl}{ }^{\circ}$ & $\operatorname{Vlou} u^{\circ} 1 a^{\circ}$ & $100^{\circ}$ & lou & le i \\
\hline Wenshui & VCV不C & $\mathrm{VClau}$ & & lau3 & lau & li a \\
\hline \multicolumn{7}{|c|}{ Region de Jinzhong } \\
\hline Yuxian & $V C$ a $V$ 不 $C$ & $\mathrm{VCl}>3$ & & 103 & 103 & $\operatorname{liE}$ \\
\hline Shouyang & $V C \propto V$ 不 $C$ & $v C l o 3$ & & 103 & 103 & le \\
\hline Taigu & VClou3v不C & VClau3 & Vlau3 & lau3 & lau 3 & li e \\
\hline Jiexiu & $v C l o u 3$ & $\mathrm{VCl}$ ou3 & & & $l o u^{(3)}$ & la \\
\hline Qixian & VClau 3 & $\mathrm{VClau3}$ & 能Vlau 3 & lau3 & $\operatorname{la} 43$ & $1 \mathrm{i}$ \\
\hline Pingyao & VCloV不C & $\mathrm{VCl} 0^{\circ}$ & $V 103^{\circ}\left(10^{\circ}\right)$ & $10^{\circ}$ & $10^{\circ}$ & la \\
\hline \multicolumn{7}{|c|}{ Region du S.E. } \\
\hline Xiangyang & $8 \quad V C V$ 不 $C$ & 能VClau & & $\operatorname{lav} u^{\circ}$ & $\operatorname{lau}^{\circ}$ & la \\
\hline Wuxiang $v$ & VClov不Cla & $\mathrm{VCl} 0^{\circ}$ & & $10^{\circ}$ & $10^{\circ}$ & $1 i z$ \\
\hline Qinxian & VClov不C & $V C 10^{\circ}$ & $V 10^{\circ} 10^{\circ}$ & $10^{\circ}$ & $10^{\circ}$ & $1 x$ \\
\hline Changzhi & VCV不C & $\mathrm{VCl}{ }^{(3)}$ & $V 10310^{\circ}$ & $10^{(3)}$ & $10^{(3)}$ & la \\
\hline Lucheng & VCV不C & veluə? & Vlua lua? & & & $1 \mathrm{a}$ \\
\hline Lingchuan & n rev不C & $v \mathrm{Cl}$ a $\mathrm{u}^{\circ}$ & $V I a u^{\circ}\left(1 \partial u^{\circ}\right)$ & la $u^{\bullet}$ & la $u^{\bullet}$ & $1 \mathrm{a}$ \\
\hline$\frac{\text { HENAN }}{\text { Huojia }}$ & VCV不C & $\mathrm{rClou}{ }^{\circ}$ & Vlau3 $10 u^{\circ}$ & lau3 & $10 u^{\circ}$ & la? \\
\hline HEBEI & & & & & & \\
\hline Chengde & VClios a & VClio3 & $V 1 \partial^{\circ}$ li o3 & lio3 & la & la \\
\hline Zunhua & VCliaulloư不: & VCliau3lou & Vliaus $100^{\circ}$ & liau3 & 兒 & 10 \\
\hline Changli & VCV不C & veli ou ${ }^{\circ}$ & Vliaus liou ${ }^{\circ}$ & liaus & {$\left[1(\mathrm{i}) \mathrm{ou}^{\circ}\right]$} & lie \\
\hline Huailu & $V \mathrm{Cl} 10^{\circ} \mathrm{pz}$ & $\mathrm{VCl} 0^{\circ}$ & $V 1$ iso $10^{\circ}$ & $1 \mathrm{ios}$ & $10^{\circ}$ & $1 x$ \\
\hline $\begin{array}{l}\text { Xianxian } \\
\text { SHANDONG }\end{array}$ & VCleao 0 & VC leao & Vleao laolao & leao & leao & leao \\
\hline Lijin & $\operatorname{VCli} i^{\bullet} \mathrm{i}^{\bullet} \mathrm{x}$ & $\operatorname{VCli}(1 \mathrm{i})$ & $\mathrm{Vli} \mathrm{i}^{\bullet} \mathrm{li}^{\bullet}$ & 1 is 3 & $1 i^{\circ}$ & la \\
\hline Dezhou Vo & $V C(1 i 0)$ liou 吧 & $v d(1$ is $)$ liouc & Vliosliou & 1 i o3 & {$\left[l(i) \circ u^{\circ}\right]$} & lia \\
\hline Linging & VClo巴 & $\operatorname{vecl} 0^{\circ}$ & Vlios $10^{\circ}$ & 1 i 3 & $\left.110^{\circ}\right)$ & $1 a$ \\
\hline Liaocheng & g VClau不 & VClau & $V 了$ la $u^{\circ}$ & 3 & lau & $1 \partial / 1 a$ \\
\hline Shanxian & (能) VClopo & $\mathrm{VCl} 0^{\circ}$ & $\operatorname{Vliau3} 10^{\circ}$ & liau 3 & $10 /$ 酛 & $1 \mathrm{a}$ \\
\hline Feicheng & VCV不C & $\mathrm{VCl} 0^{\circ}$ & & & $10^{\circ}$ & li a \\
\hline Jinan & VClo 吧 & $\operatorname{vc}(1$ i 33$) 10$ & V1io3 10 & lis3 & & \\
\hline Boshan & $v C l i 0^{\circ}$ a bo & $\mathrm{VCl}$ is ${ }^{\circ}$ & $\operatorname{Vli} 0^{\circ}$ & $1 \mathrm{is}^{\circ}$ & {$\left[1 \mathrm{io}^{\circ}\right]$} & $\operatorname{lix}$ \\
\hline Pingy i & $V \mathrm{Clau}{ }^{\circ} \mathrm{Pau}^{\circ}$ & $v \mathrm{Clau}{ }^{\circ}$ & & liau & $\mathrm{lau}^{\bullet}$ & 12 \\
\hline Zhucheng & VCV不C & $V C 10$ & & & 10 & Ia \\
\hline Pingdu & VCV不C & $\operatorname{VCl} \partial^{\circ}$ & Vlio3 la & 1 i 33 & {$\left[1 \partial^{\bullet}\right]$} & la \\
\hline
\end{tabular}

[N.B.] [ ]: il existe une variante assyllabique et/ou de type 几 化. 